\title{
Autosomal dominant thoracolaryngopelvic dysplasia: Barnes syndrome
}

\author{
J BURN, C HALL, D MARSDEN, AND D J MATTHEW \\ From The Hospital for Sick Children, Great Ormond Street, London WC1N 3JH.
}

SUMmARY We review a family in which a mother and two of her three children suffered a distinct syndrome of thoracic dystrophy with small chest volume, laryngeal stenosis, normal stature with variable asymmetry, asthenic build, and a small pelvis. In addition to the different inheritance pattern, this syndrome is distinguished from the better known Jeune syndrome by the differing thoracic and pelvic configuration.

Jeune syndrome ${ }^{1}$ is a well recognised form of autosomal recessive thoracic dystrophy characterised by lung hypoplasia and respiratory failure, associated with severe shortening of the ribs. Short stature usually becomes apparent with age and renal failure associated with cystic tubular dysplasia or glomerular sclerosis is a frequent late complication. Postaxial polydactyly of all four limbs and retinal degeneration are occasional features, while characteristic radiological features are hypoplastic iliac wings with a medial iliac spur, the latter disappearing with age. Cone shaped epiphyses develop during childhood.

In 1969, Barnes et al ${ }^{2}$ reported two unrelated infants with thoracic dystrophy. The second child showed the classical features of Jeune syndrome, but the first was unusual in that the rib shortening was less severe, there was laryngeal stenosis, and similar, though less severe, clinical features were apparent in the mother. Subsequently, this mother gave birth to a second child affected by a similar syndrome. In view of the early death of the first sib, surgery was carried out on the second in an attempt to increase the thoracic volume. This intervention was a qualified success and was the subject of a second report. ${ }^{3}$

In 1983, Bankier and Danks ${ }^{4}$ reported a thoracicpelvic dysostosis in a mother and her son, suggesting that this was a distinct autosomal dominant disorder. These authors made reference to the first paper by Barnes $e t a l^{2}$ as the only other report of apparent dominant transmission, but suggested that the presence of laryngeal stenosis and the severity of the disorder distinguished the two families.

The second affected sib was readmitted recently

Received for publication 10 June 1985

Accepted for publication 14 June 1985.

in respiratory failure at 14 years of age and the original diagnosis of Jeune syndrome was questioned. We present a reassessment of this family.

\section{Case reports}

\section{3 (FIG 1)}

This lady was born in 1941, the first child of healthy unrelated Austrian parents. She suffered several chest infections in early childhood, but subsequently grew to an above average adult height of $170 \cdot 2 \mathrm{~cm}$. She was noted to have an abnormally small pelvis at obstetric assessment during her first pregnancy, and elective caesarian section was carried out at 38 weeks. Anaesthesia was complicated by difficulty in intubation due to laryngeal stenosis and reduced lung volume associated with a small thorax. Subsequent examination revealed her to have a small chest in proportion to her height with a wide

II

1
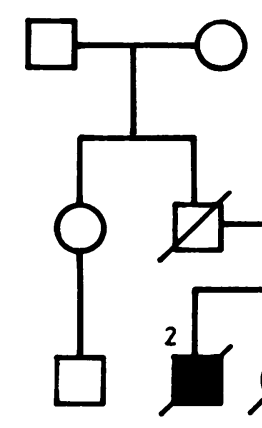

* Considered normal although not available for examination

FIG 1 Family pedigree. 
subcostal angle, horizontal ribs, and bilateral pleural thickening. She was noted to have a contracted pelvis with small, 'squared' iliac bones. Respiratory function tests showed reduced lung volumes. ${ }^{2}$ At the age of 43 she was without significant clinical problems, though she noted that she had never been able to run easily and had found big meals made her uncomfortable. Her left leg was slightly longer than the right, causing a minor degree of pelvic tilt.
Repeated radiological examination revealed a slightly narrow thoracic cage with flattened diaphragm and unusually prominent muscle attachments (fig 2a), narrowing of the pelvis and iliac bones, and an unusual flare of the iliac crest (fig $2 b$ ). In the spine, there was a thoracic scoliosis convex to the left with disc space narrowing, minor vertebral end-plate irregularity, and some spondylotic change (fig 2c).

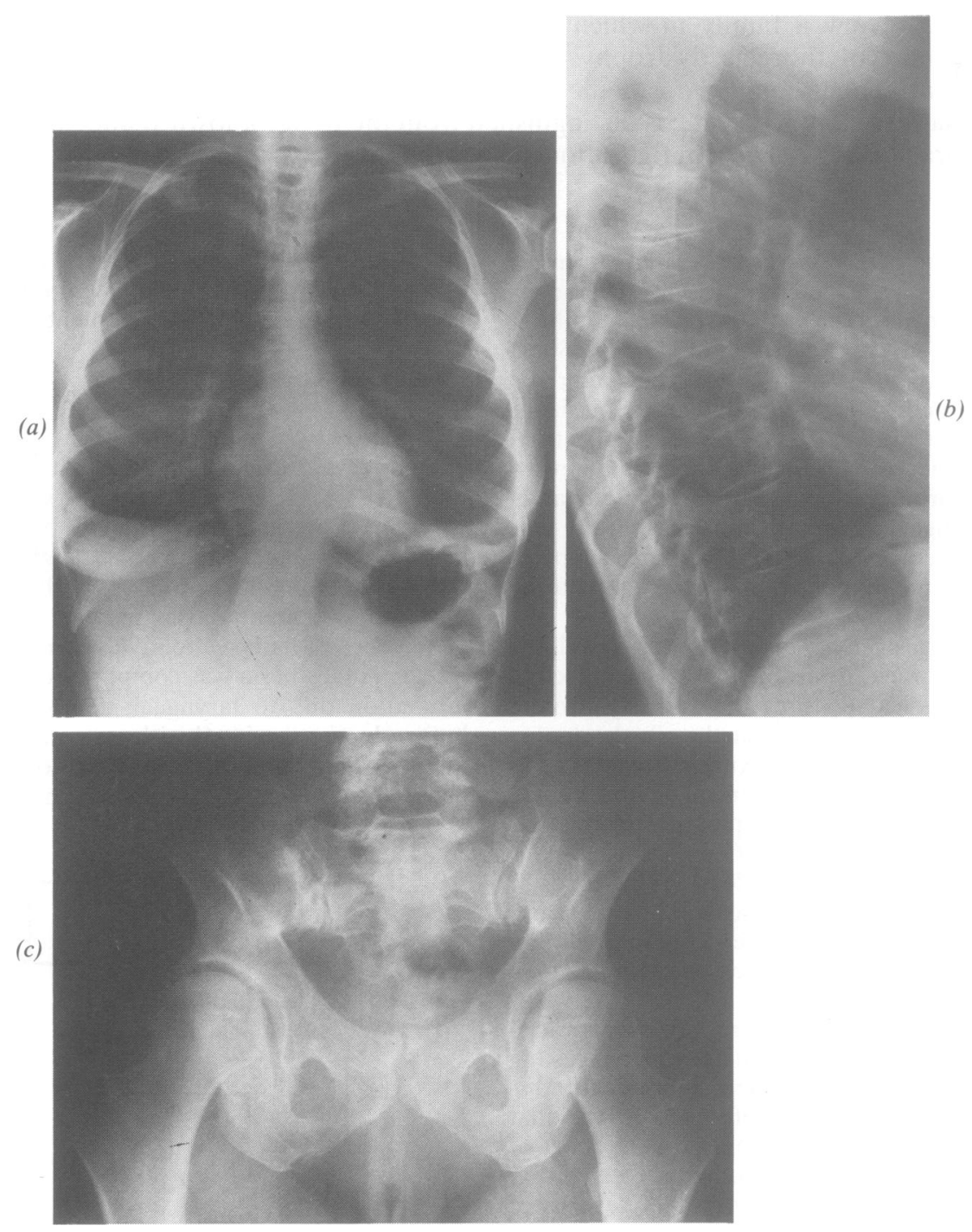

FIG 2 Radiograph of mother (II.3). (a) The thoracic cage is slightly narrow and the diaphragm is flattened with unusually prominent muscle attachments. There is scoliosis convex to the left. (b) Disc space narrowing, minor vertebral end-plate irregularity, and some spondylotic changes are evident. (c) The pelvis and iliac bones are narrow, but there is an unusual flare of the iliac crest. The obturator foramen is relatively small. 
(a)
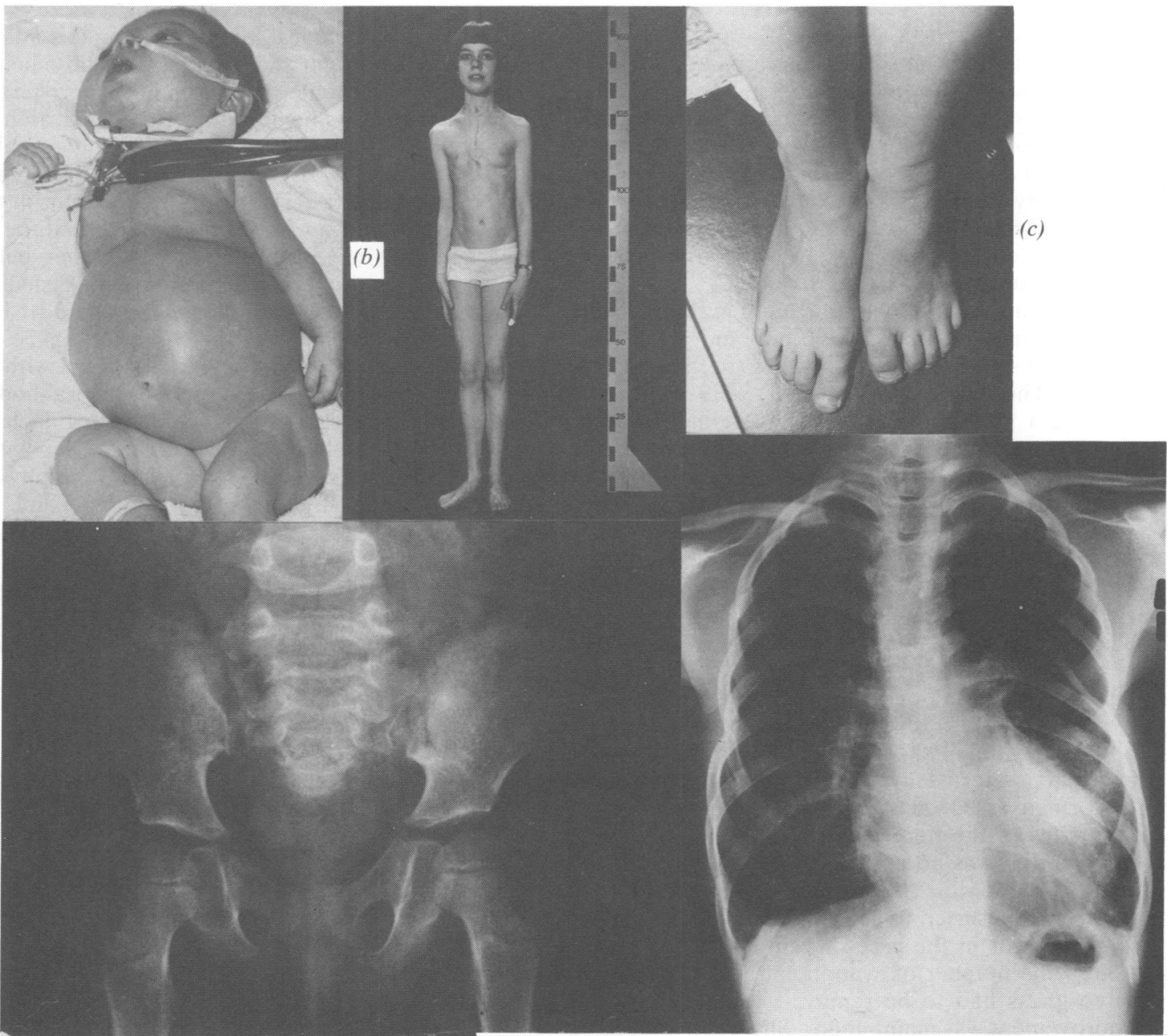

(d)

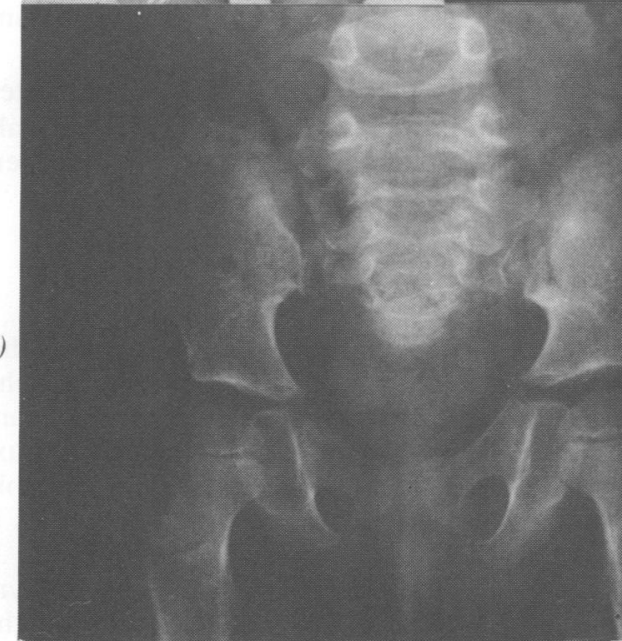

FIG 3 Clinical and radiological features of second affected child (III.3) in infancy and adolescence. (a) Physical appearance at 4 months of age. (b) Physical appearance at 14 years of age: not dysmorphic, asthenic build with mild asymmetry of limb length. (c) Asymmetry of feet. (d) Pelvis: reduction in all dimensions with shallow sacroiliac notch and small iliac wings. (e) AP thorax at 15 years. Thoracic cage remains narrow and lung volume small. A mild double scoliosis is evident. (f) Lateral thorax at 7 years 8 months. The vertebral bodies have an unusual configuration with convex vertebral end-plates and narrow disc spaces. The spine is unusually straight with loss of normal dorsal kyphosis. There are residual wire sutures following surgical intervention in infancy. 
III.2 (FIG 1) (GOS 404646)

The first child in the sibship was a male born at 38 weeks' gestation, weighing $2.5 \mathrm{~kg}$. His very small thorax was immediately obvious at birth. Ventilatory support was complicated first by difficulty in intubation due to narrowing of the larynx, and later by a very high resistance to inflation. It was suspected that significant lung hypoplasia was present so no surgical intervention was considered and death occurred at 7 weeks of age.

At necropsy, inflation of the lungs in situ revealed their volume to be only $30 \mathrm{ml}$ and they were somewhat small, weighing only $43 \mathrm{~g}$ (normal for age, $60 \mathrm{~g})$. The laryngeal cartilages were abnormal, forming a 'funnel' resulting in severe narrowing of the first part of the trachea below the level of the cricoid cartilage. The costochondral junctions were widely expanded and showed marked dystrophic changes histologically, but otherwise the skeleton was unremarkable.

III.3 (FIG 1) (GOS 421881)

The second sib, a female, was born at 38 weeks' gestation by elective caesarian section, weighing $2 \cdot 3$ kg. Pregnancy had been normal, but an $x$-ray at 34 weeks' gestation revealed the fetus to have an abnormally small thorax. Ventilatory support was necessary after delivery and tracheostomy was performed at 33 days (fig 3a).

In view of the findings in the first sib, operative intervention was attempted. In March 1969, at the age of $6 \frac{1}{2}$ months, the sternum was split and bone grafts were inserted to act as struts, with a resultant improvement in the ease of ventilation and reduction in the number of cyanotic attacks. Two of the three grafts had to be removed subsequently, but improvement was maintained. ${ }^{3}$ She was managed on home ventilation from the age of 18 months to $41 / 2$ years, though she suffered repeated admissions for the treatment of respiratory infections and pneumothoraces. She eventually became independent of ventilatory support at 4 years and her tracheostomy tube was removed at the age of 9 years. Subglottic stenosis was present at this stage though it was not possible to distinguish whether this was primary or secondary to neonatal intubation. In view of the family history the former is more likely.

In 1982 , at the age of 14 years, she was managing successfully in normal school and was assessed as being approximately one year behind her peers in academic achievement. In July 1983, she was readmitted in respiratory failure following a chest infection and a tracheostomy tube was reinserted. Over the subsequent three months she succeeded in becoming independent of the ventilator once more and was discharged.
Review of her clinical features during this admission revealed a bright and cheerful girl whose height was on the 50th centile. She was not dysmorphic (fig $\overrightarrow{0}$ 3 b) and was of asthenic build with proportionate $\frac{\bar{\sigma}}{\sigma}$ limb lengths, though careful comparison revealed $\overline{\bar{\sigma}}$ slight asymmetry with the right limbs being slightly larger than the left. This was more apparent in the feet where there was, in addition, a long first toe on $\omega$ the right foot (fig 3c). Radiologically, her pelvis $\vec{\circ}$ showed a reduction in all dimensions with a shallow $\vec{\overrightarrow{ }}$ sacroiliac notch and small iliac wings (fig 3d). Her $\vec{\omega}$ thorax remained narrow with short ribs (figs $3 e, f$ ). In early childhood her spine was abnormal with an immature oval configuration of the vertebral bodies and narrow disc spaces in the dorsal region later i progressing to scoliosis. Radiographs of her hands revealed no evidence of cone shaped epiphyses. $\bullet$ Plasma biochemistry, renal ultrasound, and in- 음 travenous pylogram showed no evidence of im- $-\overrightarrow{-}$ paired kidney function.

Following a period of stability she was readmitted in severe respiratory failure and cor pulmonale which led to her death. Necropsy was not performed.

\section{Discussion}

This review confirms the earlier impression that this family suffers from a distinct thoracic dystrophy syndrome, characterised by autosomal dominant inheritance, a small, rigid, 'bell shaped' thorax, laryngeal stenosis, and a small pelvis. The variable expression in the three affected family members is typical of a dominant disorder. The syndrome is easily distinguished from the recessive Jeune syndrome by the different thoracic configuration, the $\frac{\sigma}{3}$ presence of laryngeal stenosis, the absence of iliac spurs in infancy, and later the absence of renal $\delta$ involvement or coned epiphyses. Other distinctions 3 are the bony changes in the spine, normal stature, $O$ and absence of polydactyly.

It remains to be established whether, as the authors suggest, the family reported by Bankier and $\bar{N}$ Danks ${ }^{4}$ constitutes a distinct syndrome. Given the or variation observed in the family reported here it is $\mathrm{O}$ entirely possible that the mother and son reported $N$ from Australia have a milder expression of what $\bar{x}$ should be called thoracolaryngopelvic dysplasia or Barnes syndrome.

This work was performed while Dr Burn was

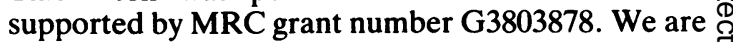
grateful to Mrs Melanie Barham and Mrs June $\stackrel{\mathbb{D}}{2}$ Gadsby for the preparation of the manuscript. 


\section{References}

' Jcunc M. Bcraud C. Carrou R. Dystrophie thoracique asphyxiante de caractère familial. Arch Fr Pediatr 1955:12:886.

2 Barnes ND. Hull D. Symons JS. Thoracic dystrophy. Arch Dis Child 1969:44:11-7.

${ }^{3}$ Barnes ND, Hull D. Milner AD, Waterston DJ. Chest reconstruction in thoracic dystrophy. Arch Dis Child 1971;46: 833-7.
4 Bankier A, Danks DM. Thoracic-pelvic dysostosis; a 'new' autosomal dominant form. J Med Genet 1983;20:276-9.

Correspondence and requests for reprints to Dr J Burn, Regional Genetics Advisory Service, 19 Claremont Place, Newcastle upon Tyne NE2 4AA. 